\title{
Efeito de quatro dias consecutivos de jogos sobre a potência muscular, estresse e recuperação percebida, em jogadores de futsal
}

CDD. 20.ed. 796.07

796.33

\author{
Victor Hugo FREITAS" \\ Eberton Alves de SOUZA** \\ Ricardo Santos OLIVEIRA* \\ Lucas Adriano PEREIRA* \\ Fábio Yuzo NAKAMURA*
}

*Centro de Educação Física e Esporte, Universidade Estadual de Londrina.

${ }^{* *}$ Faculdade Integrado de Campo Mourão.

\section{Resumo}

0 objetivo desse estudo foi analisar o efeito de jogos de futsal realizados em dias consecutivos sobre 0 desempenho em testes de saltos verticais e sobre o estresse e a recuperação de atletas dessa modalidade. Participaram deste estudo 11 atletas do sexo masculino $(24,3 \pm 5,0$ anos, 1,73 $\pm 0,07 \mathrm{~cm}, 75,7 \pm 9,0$ $\mathrm{kg}, 11,2 \pm 4,1 \%$ de gordura), pertencentes a uma equipe de futsal. A equipe foi monitorada durante a fase final dos Jogos Abertos do Paraná, com jogos realizados em quatro dias consecutivos. 0 RESTQSport foi aplicado antes e após a competição, e os testes de "squat jump" (SJ) e de salto vertical com contramovimento (CMJ) foram realizados na manhã de todos os dias de jogos. $0 \mathrm{SJ}$ apresentou uma provável diminuição $(0 / 34 / 66 \%)$ antes do segundo jogo comparado com antes do primeiro, uma muito provável diminuição (0/3/97\%) antes do terceiro jogo comparado com antes do primeiro e uma provável diminuição (2/20/78\%) antes do quarto jogo comparado com antes do primeiro. $0 \mathrm{CMJ}$ apresentou uma provável diminuição antes do segundo (0/19/81\%), terceiro (3/20/77\%) e quarto jogo (1/10/89\%) em relação à antes do primeiro. A escala recuperação física do RESTQ-Sport foi menor no pós-competição, comparada com o pré-competição $(p<0,05)$ e a escala fadiga foi maior no pós-competição comparada com pré-competição $(p<0,05)$. A diferença entre o $\Sigma$ das escalas de recuperação e $0 \Sigma$ das escalas de estresse no pós-competição $(7,5 \pm 9,8)$ foi menor que o pré-competição $(9,8 \pm 9,1 ; p=0,03)$. Em conclusão, o decréscimo no desempenho de saltos verticais e as alterações deletérias nas escalas do RESTQ-Sport sugerem que houve acúmulo de fadiga ao longo de jogos de futsal realizados em dias consecutivos.

Palavras-Chave: Esportes; Desempenho atlético; Fadiga; Dano muscular.

\section{Introdução}

O futsal é uma modalidade esportiva de natureza intermitente com esforços de alta intensidade e curta duração $(2-3 \mathrm{~s})$, intercalados com breves períodos de recuperação (20-30 s) ${ }^{1}$, somados a constantes mudanças de velocidade e direção ${ }^{1-2}$. Dessa forma, durante a partida há uma elevada exigência dos sistemas aeróbio e anaeróbios para o suprimento de energia ${ }^{1}$. Tais exigências metabólicas levam a uma elevada frequência cardíca média ( $-90 \%$ da frequência cardíaca máxima) durante as partidas ${ }^{1-3}$, as quais são disputadas em dois tempos cronometrados de 20 minutos podendo, dessa forma, uma partida ter a duração real de aproximadamente 80 minutos $^{3-4}$.
No Brasil e em outros países, equipes dessa modalidade podem disputar de dois a quatro jogos por semana, realizados em dias consecutivos em competições de menor duraçãa ${ }^{5-6}$, como por exemplos os Jogos Abertos do Interior. A alta intensidade das partidas de futsal provoca dano muscular, inflamaçãa ${ }^{7}$ e redução da função muscular ${ }^{5}$. Dessa maneira, apesar da necessidade de níveis adequados de recuperação para um bom desempenho nas partidas $^{8}$, o intervalo de tempo entre jogos realizados em dias consecutivos pode ser insuficiente para promover uma recuperação adequada aos jogadores. Em outros esportes coletivos, foi demonstrado que 
atletas de basquetebol, por exemplo, apresentavam fadiga acumulada refletida no menor desempenho em teste de salto vertical durante um campeonato com jogos em dias consecutivos? ${ }^{9}$. Resultados similares também foram encontrados em jogadores de futebol ${ }^{10}$. No entanto, essa problemática não foi investigada no futsal durante um campeonato com essas características.

Os sintomas de fadiga observados após exercícios intensos são refletidos em marcadores psicofisiológicos ${ }^{8}$ e no desempenho em testes físicos? ${ }^{9}$. Dessa forma, testes de desempenho, bem como questionários com indicadores psicológicos específicos para o esporte, são boas estratégias para avaliar fadiga ocasionada por exercícios intensos ${ }^{8,11}$. Neste sentido, o teste de desempenho no salto vertical se mostra sensível para identificar a fadiga após partidas de futsal ${ }^{5}$ e rúgbi ${ }^{12}$. A diminuição da função muscular observada a partir de testes de salto vertical é relacionada com a fadiga metabólica e neuromuscular e com o dano muscular ocasionado pelo exercício, podendo permanecer por vários dias ${ }^{12-14}$. Já o Questionário de Estresse e Recuperação para atletas (RESTQ-Sport) ${ }^{15-16}$ é

\section{Método}

\section{Amostra}

Participaram deste estudo 11 atletas de futsal do sexo masculino, com idade de 24,3 $\pm 5,0$ anos, estatura de $1,73 \pm 0,07 \mathrm{~cm}$, peso corporal de 75,7 $\pm 9,0 \mathrm{~kg}$ e percentual de gordura de $11,2 \pm 4,1 \%$, pertencentes a uma equipe de alto nível da categoria adulto da primeira divisão estadual. Foram adotados como critérios de inclusão: pertencer à equipe em questão e participar por um período de no mínimo cinco minutos, contínuos ou não, em todos os jogos observados neste estudo. $\mathrm{O}$ atleta poderia retornar à quadra por mais de uma vez de acordo com a opção tática do treinador. $\mathrm{O}$ rodízio entre os jogadores durante uma partida é comum no futsal, pois a demanda física do jogo é muito alta. O tempo de permanência em quadra de cada atleta foi cronometrado manualmente por um membro da comissão técnica da equipe. Essa é uma prática regular da equipe, uma vez que a comissão técnica faz esse registro e acompanhamento em todos os jogos durante a temporada. Foram adotados como critérios de exclusão: apresentar lesões mioarticulares e/ou participar por menos de cinco minutos nos jogos. $\mathrm{O}$ amplamente utilizado como uma ferramenta sensível para identificar alteraçôes no balanço estresse/ recuperação de atletas de várias modalidades ${ }^{5,17-20}$. O RESTQ-Sport é uma ferramenta simples, de baixo custo operacional e de fácil aplicação, que pode fornecer informações importantes relacionadas a essas variáveis durante um campeonato com jogos realizados em dias consecutivos.

Conhecer o impacto de jogos realizados em dias consecutivos sobre o desempenho em testes físicos simples e o balanço entre estresse e recuperação em atletas de futsal é necessário, pois possibilita planejar estratégias de recuperação adequadas de maneira a preservar a integridade física dos atletas e potencializar o desempenho nos jogos. Assim, o objetivo desse estudo foi analisar o efeito de jogos de futsal realizados em dias consecutivos sobre a potência muscular em saltos verticais e o balanço entre estresse e recuperação de atletas dessa modalidade. A hipótese desse estudo é que alterações negativas no balanço entre estresse e recuperação e queda do desempenho em testes de saltos verticais ao longo dos jogos consecutivos seriam registrados.

estudo foi aprovado pelo comitê de ética em pesquisa local (parecer $n^{\circ} 220 / 10$ ) e todos os procedimentos do estudo foram esclarecidos aos atletas, que assinaram um termo de consentimento livre e esclarecido.

\section{Procedimentos}

A equipe de futsal foi monitorada durante a fase final dos Jogos Abertos do Paraná, com jogos realizados diariamente durante quatro dias consecutivos. Todos os jogos foram realizados no período da noite (entre 19 e 22 horas). O RESTQ-Sport foi aplicado na manhã do primeiro dia de competição e na manhã seguinte ao último dia de competição. Os testes de desempenho "squat jump" (SJ) e "countermovement jump" (CMJ) foram realizados, nessa ordem, em todos os dias de jogos, no período da manhã, entre oito e nove horas.

\section{Testes de saltos verticais}

Os testes de saltos verticais, SJ e CMJ, foram realizados em todos os dias de jogos, entre oito e nove horas da manhã, com os atletas alimentados. 
Os testes de saltos verticais foram realizados em uma quadra poliesportiva, localizada nas dependências onde os atletas estavam alojados. Antes do início dos testes, os atletas realizaram um aquecimento com a duração de cinco minutos. $\mathrm{O}$ aquecimento foi padronizado pelo preparador físico da equipe como descrito a seguir: corridas em baixa intensidade, saltos submáximos e exercícios de alongamento.

O teste SJ foi utilizado para avaliar a potência de membros inferiores, minimizando a influência de componentes elásticos e reflexo de estiramento. $\mathrm{O}$ teste CMJ foi utilizado para avaliar a potência de membros inferiores com participação de componentes elásticos e reflexo de estiramento. A altura dos saltos foi medida por meio de um tapete de contato (Hidrofit ${ }^{\circledR}$, Brazil), interligado a um computador com programa específico (Hidrofit ${ }^{\circledR}$, Brazil). Foram realizados três saltos de cada modalidade de salto, com 30 s de intervalo entre eles. Para a realização do SJ, os jogadores iniciaram o teste na posição agacha$\mathrm{da}$, por pelo menos $3 \mathrm{~s}$, com as mãos no quadril e joelhos flexionados a um ângulo de $\sim 90^{\circ}{ }^{21}$. Desse modo, o $S J$ não apresentou a fase excêntrica e não foi permitido ao atleta realizar contramovimento. No CMJ, os jogadores iniciaram o teste na posição ortostática, com as mãos no quadril, flexionaram os joelhos a um ângulo autosselecionado, e executaram a fase concêntrica com a maior potência possível. Desse modo, o CMJ foi executado com a utilização do ciclo alongamento-encurtamento. Não foi permitido o auxílio dos membros superiores em ambos os saltos. Adotou-se como medida de desempenho a maior altura das três tentativas computadas. Os saltos apresentam alta reprodutibilidade, com o coeficiente de correlação intraclasse de 0,97 para ambos, e coeficiente de variação de $2,4 \%$ e $2,6 \%$ para o SJ e CMJ, respectivamente ${ }^{22}$.

Questionário de estresse e recuperação para atletas (RESTQ-Sport)

O RESTQ-Sport foi aplicado no primeiro dia de competição, no período da manhã, antes dos testes de saltos verticais, e na manhã seguinte ao último jogo da equipe no campeonato, entre oito e nove horas. Os atletas preencheram os questionários ao mesmo tempo, de maneira individual, no quarto no qual os mesmos estavam alojados. Os membros da comissão técnica acompanharam o preenchimento dos questionários para que os atletas não conversassem uns com os outros durante o preenchimento. Os atletas eram habituados com o questionário, uma vez que o preparador físico o utilizava para monitoramento do estresse e recuperação dos atletas semanalmente.

O RESTQ-Sport ${ }^{15}$, versão em língua portugue$\mathrm{sa}^{16}$, foi utilizado para avaliar o estresse e a recuperação dos atletas referentes às atividades dos últimos três dias e três noites. Esse questionário é composto por 76 questōes, com respostas em escala tipo Likert de 0 (nunca) a 6 (sempre). As questōes são divididas em 19 escalas, 10 relacionadas ao estresse e nove relacionadas à recuperação. Calculou-se a soma das escalas relacionadas ao estresse $(\Sigma \mathrm{E})$ e à recuperação $(\Sigma \mathrm{R})$, bem como a diferença entre $\Sigma \mathrm{R}$ e $\Sigma \mathrm{E}$.

\section{Análise estatística}

Testou-se a distribuição dos dados por meio do teste de Shapiro-Wilk. A diferença nas escalas do RESTQ-Sport pré- e pós-competição foi analisada pelo teste de Wilcoxon. O programa utilizado foi o Statistica (v.8.0, StatSoft ${ }^{\circledR}$, Tulsa, Ok), considerando uma probabilidade de erro tipo I $(\alpha)$ de $5 \%$. Para a comparação do desempenho no $\mathrm{SJ}$ e no CMJ, os dados foram transformados em logaritmo natural e a diferença percentual em relação ao jogo antecedente $(\Delta \%)$ foi analisada mediante inferência prática baseada em magnitudes ${ }^{23}$. Para isso, foi estimada a mínima mudança detectável percentual $(0,20$ multiplicado pelo desvio padrão inicial baseado no tamanho de efeito) e calculada a chance do efeito observado ser considerado como aumento, estabilização ou diminuição no desempenho. A mudança foi avaliada qualitativamente como se segue: $<1 \%$ quase certamente não; $1-5 \%$ muito improvável; 5-25\% improvável; $25-75 \%$ possível; $75-95 \%$ provável; $95-99 \%$ muito provável e > 99\% quase certamente sim. Se os valores negativos e positivos apresentassem resultados $>10 \%$, a inferência era considerada inconclusiva. As análises foram realizadas através das planilhas disponíveis em http:// sportsci.org/resource/stats/. 


\section{Resultados}

A variável analisada para avaliar o desempenho foi altura dos saltos verticais. Os resultados referentes ao desempenho no SJ são apresentados na FIGURA 1. A análise qualitativa apresentou uma possível diminuição (aumento/estabilização/diminuição = 0/34/66) da altura de salto obtida antes do segundo jogo comparada com o obtido antes do primeiro, muito provável diminuição $(0 / 3 / 97)$ no desempenho obtido previamente ao terceiro jogo comparado com o obtido prévio ao primeiro e uma provável diminuição $(2 / 20 / 78)$ no desempenho observado previamente ao quarto jogo comparado com o observado prévio ao primeiro jogo.

Os resultados referentes ao desempenho no CMJ são apresentados na FIGURA 2. A análise qualitativa dos dados mostra que o desempenho antes do segundo $(0 / 19 / 81)$, terceiro $(3 / 20 / 77)$ e quarto jogos (1/10/89) apresentou uma provável diminuição em relação ao desempenho antes do primeiro jogo. A TABELA 1 apresenta os valores de média e desvio padrão dos resultados obtidos para o $\mathrm{SJ}$ e $\mathrm{CMJ}$ ao longo dos jogos consecutivos.
*Provável diminuição (0/34/66);

***Provável diminuição (2/20/78);

**Muito provável diminuição (0/3/97).
*Provável diminuição (0/19/81);

**Provável diminuição (3/20/77);

***Provável diminuição (1/10/89).

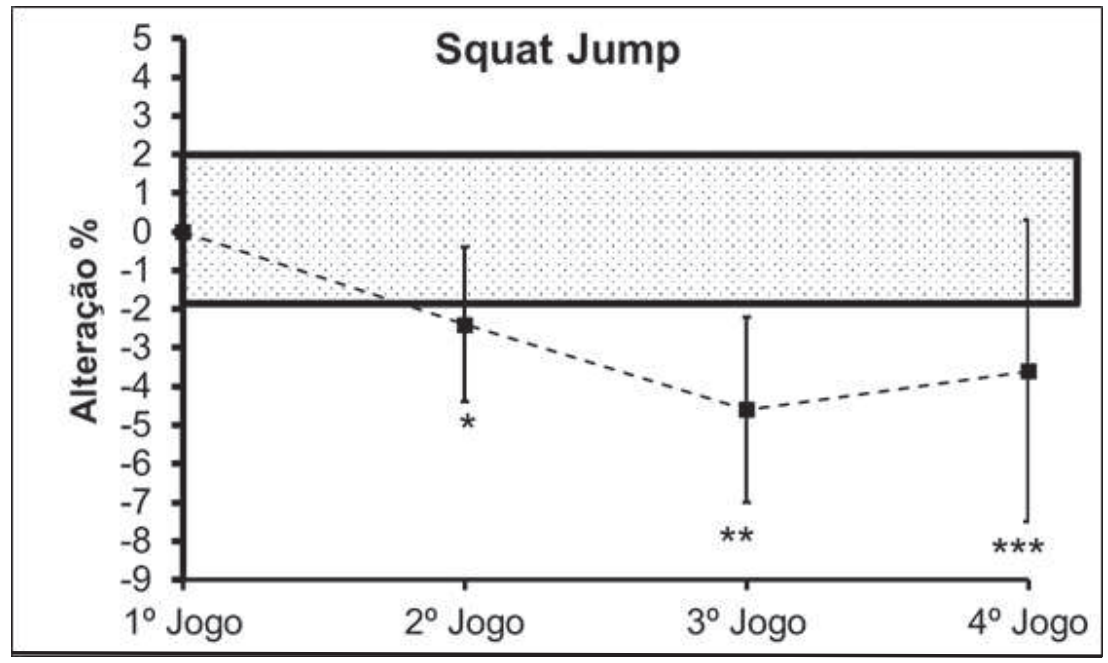

FIGURA 1 - Alteração \% na altura alcançada no "Squat Jump”.

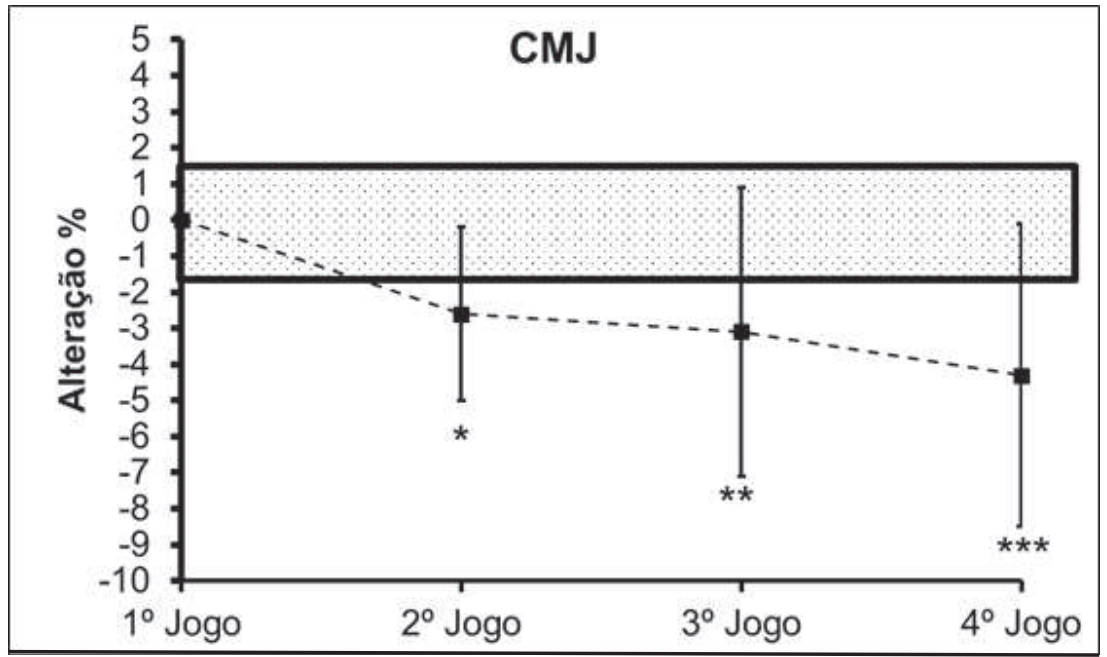

FIGURA 2 - Alteração \% na altura alcançada no CMJ. 
TABELA 1 - Média e desvio padrão do desempenho obtido no SJ e CMJ ao longo dos jogos consecutivos.

\begin{tabular}{|c|c|c|c|c|c|c|c|}
\hline \multicolumn{4}{|c|}{ SJ } & \multicolumn{4}{|c|}{$\mathrm{CMJ}$} \\
\hline $1^{\circ}$ Jogo & $2^{\circ}$ Jogo & $3^{\circ}$ Jogo & $4^{\circ}$ Jogo & $1^{\circ}$ Jogo & $2^{\circ} \mathrm{Jogo}$ & $3^{\circ}$ Jogo & $4^{\mathrm{o}}$ Jogo \\
\hline $38,6 \pm 3,5$ & $37,7 \pm 3,3$ & $36,9 \pm 3,6$ & $37,3 \pm 3,2$ & $46,1 \pm 3,0$ & $45 \pm 3,7$ & $44,9 \pm 4,7$ & $44,2 \pm 3,7$ \\
\hline
\end{tabular}

Conforme descrito na FIGURA 3, apenas as escalas recuperação física e lesões do RESTQ-Sport foram diferentes quando comparados os resultados pós- e pré-competição. A escala recuperação física foi menor no pós-competição, comparada com o observado antes da competição $(p<0,05)$ e a escala fadiga foi maior no pós-competição comparada com pré-competição ( $p$ $<0,05)$. O $\Sigma \mathrm{R}$ e o $\Sigma \mathrm{E}$ não foram diferentes quando comparado pré e pós-competição $(\mathrm{p}>0,05)$. No entanto, nota-se que a diferença entre $\Sigma \mathrm{R}$ e $\Sigma \mathrm{E}$ no póscompetição $(7,5 \pm 9,8)$ foi menor quando comparado com o pré-competição $(9,8 \pm 9,1 ; \mathrm{p}=0,03)$.

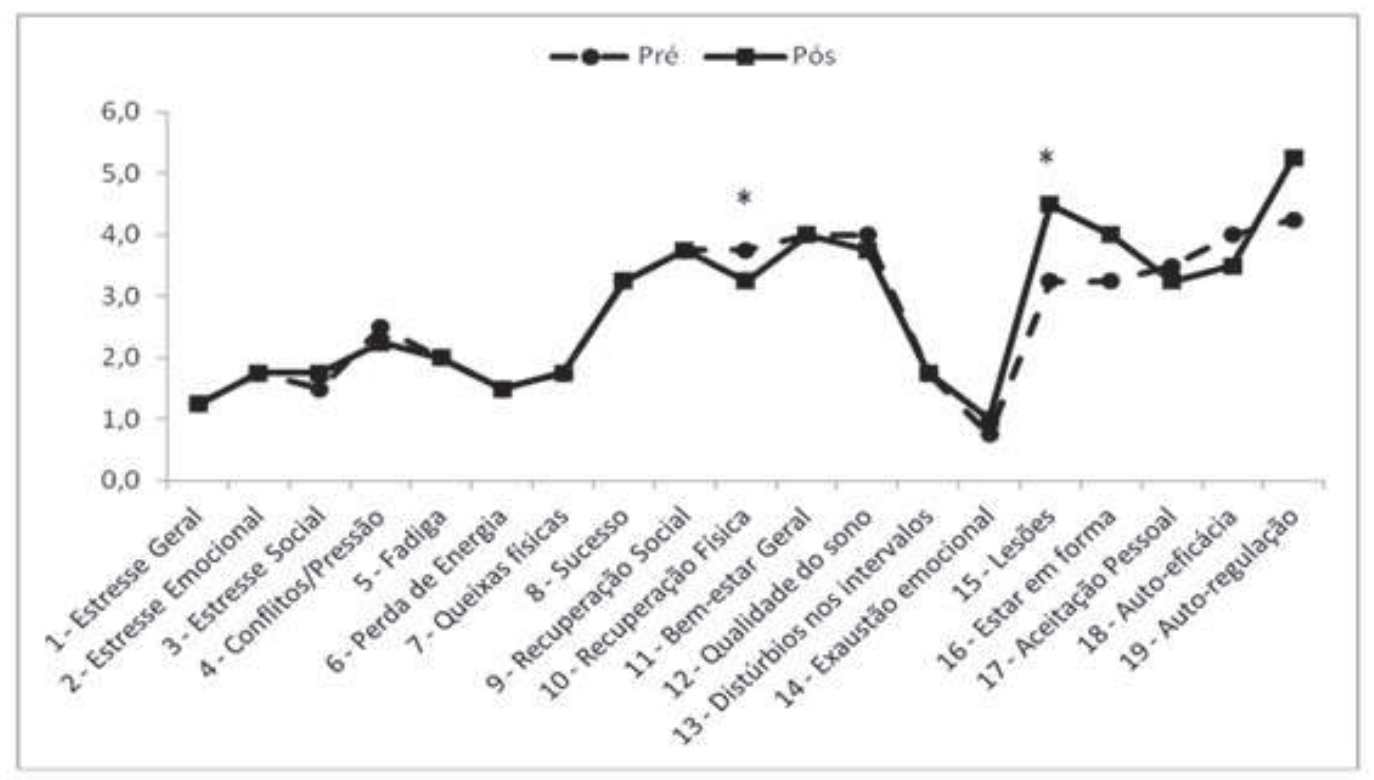

*diferença significativa

FIGURA 3 - Mudanças nas escalas do RESTQ-Sport pré e pós campeonato.

\section{Discussão}

As principais constatações deste estudo foram que a altura alcançada nos testes $\mathrm{SJ}$ e no CMJ diminuíram ao longo da competição. Além disso, houve uma diminuição no escore do RESTQ-Sport relacionado à recuperação física e um aumento no escore relacionado a lesões pós-competição comparado com o momento pré-competição. É possível notar também que a diferença entre o somatório das escalas de recuperação e o somatório das escalas de estresse foi menor pós-competição, comparado com o momento pré-competição. Esses resultados confirmam a hipótese inicial desse estudo.

Os movimentos executados pelos membros inferiores nos jogos de futsal como correr, saltar e andar utilizam, em sua maioria, açôes de ciclo alongamento-encurtamento $(\mathrm{CAE})^{13}$. Exercícios exaustivos com esse padrão de movimento podem induzir alteraçōes musculares com consequentes alterações reflexas que podem proporcionar diminuição no desempenho nos saltos verticais que envolvem o $\mathrm{CAE}^{24}$. HoriTA et al. ${ }^{25}$ apontam para uma maior sensibilidade de testes com CAE à fadiga ocasionada por exercícios dessa natureza quando comparados a testes que envolvem açôes puramente concêntricas. Por outro lado, as adaptações ocasionadas pelos constantes treinamentos específicos para a modalidade podem ter ocasionado efeito protetor do exercício repetido, refletindo em menores alteraçōes deletérias ao $\mathrm{CMJ}^{26}$. 
Assim, a sensibilidade dos testes de saltos verticais mostrou-se semelhante para identificar a ocorrência de queda da função muscular ao longo dos jogos, com uma maior magnitude observada no teste de SJ.

Embora os resultados apresentados no presente estudo não permitam apontar o mecanismo responsável pela diminuição no desempenho nos testes de salto ao longo dos dias, um dos principais fatores relacionados à queda da potência muscular nos dias seguintes aos jogos pode estar relacionado ao dano muscular ocasionado pelo exercício ${ }^{12-14}$. Nesse sentido, de Moura et al. ${ }^{7}$ demonstraram um aumento nas concentrações plasmáticas de creatina kinase (CK) e em processos inflamatórios imediatamente após uma partida de futsal. Com isso, os autores sugerem que partidas dessa modalidade podem ocasionar dano muscular. Isso, por sua vez, pode ter contribuído para a redução no desempenho nos testes de saltos nos dias subsequentes aos jogos de futsal reportados no presente estudo. Outro mecanismo que pode ter influenciado nessas respostas é a fadiga de baixa frequência ${ }^{27}$. Devido às açōes repetidas do CAE realizadas durante jogos de futsal, a fadiga de baixa frequência pode ter sido desencadeada, principalmente, por uma menor liberação do cálcio do retículo sarcoplasmático, o que por sua vez pode prejudicar o acoplamento das pontes cruzadas, diminuindo a força de contração muscular. Consequentemente, o desempenho nos testes de saltos pode ter sido comprometido ${ }^{27}$.

Outro fato importante a ser destacado é que os valores médios percentuais de queda observados nos testes de SJ e CMJ (4 e $5 \%$, respectivamente) estão próximos aos valores de queda apresentados $(-7 \%)$ no CMJ após consecutivas partidas de futebol ${ }^{28} \mathrm{e}$ handebol ${ }^{29}$. Vale ressaltar que no estudo de RowselL et al..$^{28} \mathrm{a}$ amostra foi composta por atletas juniores de futebol, e no estudo de RongLAn et al. ${ }^{29}$ a amostra foi composta de mulheres jogadoras de handebol. Desse modo, independente da modalidade esportiva ou mesmo da amostra dos estudos supracitados, parece que competições com jogos realizados em dias consecutivos provocam um percentual de queda aproximado em testes de salto vertical.
A mudança negativa das escalas recuperação física, lesōes e da diferença entre $\Sigma \mathrm{R}$ e $\Sigma$ E do RESTQ-Sport após a competição com jogos consecutivos de futsal apresentadas no presente estudo, sugerem que a competição ocasionou aumento no estresse e uma redução na percepção de recuperação dos jogadores de futsal. Essas mesmas escalas foram alteradas em outros estudos, em momentos de treinamento intensificado no rúgbi ${ }^{17}$, triatlo ${ }^{18}$ e natação ${ }^{19}$, sendo relacionadas à fadiga acumulada nesses períodos ${ }^{17-18}$. A alteração nas escalas do RESTQ-Sport no presente estudo pode ter alguma relação com a fadiga/dano muscular. No entanto, a relação entre esses dois constructos deve ser elucidado apropriadamente em estudos futuros.

Apesar das alterações na potência de membros inferiores observados nos testes de saltos verticais e as alterações de estresse e recuperação observadas pelo RESTQ-Sport sugerirem haver acúmulo de fadiga nos jogos de futsal realizados em dias consecutivos, não é possível afirmar que o desempenho dos atletas durante as partidas foi alterado. A queda do desempenho de corrida durante as partidas de futebol realizadas em dias consecutivos foi demonstrada no estudo realizado por Rowsell et al..$^{10}$, o que sugere uma proposta de futuros estudos com o futsal e outras modalidades esportivas. Além disso, estudos que investiguem os componentes fisiológicos responsáveis pelas alteraçôes no desempenho são necessários para fortalecer os resultados do presente estudo e auxiliar na escolha da estratégia de recuperação adequada.

Conclui-se que o campeonato de futsal investigado, com jogos realizados em dias consecutivos, ocasionou queda de desempenho nos testes de saltos verticais ao longo da competição e alterações deletérias nas escalas do RESTQ-Sport ao final do campeonato. Isso mostra que competiçóes esportivas organizadas com jogos em dias consecutivos pode comprometer o desempenho físico dos atletas, bem como alterar o equilíbrio entre estresse e recuperação. A sobrevida desses estados alterados, associados a correlatos fisiológicos e imunológicos, deve ser objeto de investigações futuras. 


\section{Abstract}

Effect of four successive days of games in muscle power, perceived stress and recovery in futsal players

The aim of this study was to analyze the effect of futsal games conducted on consecutive days on vertical jump performance test and stress and recovery of 11 professional players (male, $24.3 \pm 5.0$ years, $1.73 \pm$ $0.07 \mathrm{~cm}, 75.7 \pm 9.0 \mathrm{~kg}, 11.2 \pm 4.1 \%$ fat). The team was monitored during the final phase of Paraná Open Games, with games in four consecutive days. The RESTQ-Sport questionnaire was applied before and after the competition, and the squat jump (SJ) and countermovement vertical jump (CMJ) tests were performed every morning when the games were held. The SJ presented a likely decrease when comparing before the second game to before the first game $(0 / 34 / 66 \%)$ and before the fourth game to before the first game $(2 / 20 / 78 \%)$, and a very likely decrease before the third game when compared to before the first game $(0 / 3 / 97 \%)$. The CMJ showed a likely decrease before the second (0/19/81\%), third (20/03/77\%) and fourth game $(01 / 10 / 89 \%)$ compared to before the first game. The physical recovery scale of the RESTQ-Sport was lower in the post-competition compared to pre-competition $(p<0.05)$ and the fatigue scale was greater in the post-competition compared to pre-competition $(p<0.05)$. The difference between the $\Sigma$ recovery scales and the $\Sigma$ stress scales in post-competition $(7.5 \pm 9.8)$ was lower when compared to the pre-competition $(9.8 \pm 9.1, p=0.03)$. In summary, the decrease in vertical jump performance and deleterious changes in the RESTQ-Sport scales suggest a fatigue accumulation over consecutive days of futsal games.

KEY WORDS: Sports; Athletic performance; Fatigue; Muscle damage.

\section{Referências}

1. Castagna C, D'Ottavio S, Granda Vera J, Barbero Alvarez JC. Match demands of professional futsal: a case study. J Sci Med Sport. 2009;12:490-4.

2. Rodrigues VM, Ramos GP, Mendes TT, et al. Intensity of official futsal matches. J Strength Cond Res. 2011;25:2482-7.

3. Barbero-Alvarez JC, Soto VM, Barbero-Alvarez V, Granda-Vera J. Match analysis and heart rate of futsal players during competition. J Sports Sci. 2008;26:63-73.

4. Dogramaci SN, Watsford ML, Murphy AJ. Time-motion analysis of international and national level futsal. J Strength Cond Res. 2011;25:646-51.

5. Tessitore A, Meeusen R, Pagano R, Benvenuti C, Tiberi M, Capranica L. Effectiveness of active versus passive recovery strategies after futsal games. J Strength Cond Res. 2008;22:1402-12.

6. Miloski B, Freitas VH, Bara-Filho MG. Monitoramento da carga interna de treinamento em jogadores de futsal ao longo de uma temporada. Rev Bras Cineantropom Desempenho Hum. 2012;14:671-9.

7. Moura NR, Cury-Boaventura MF, Santos VC, et al. Inflammatory response and neutrophil functions in players after a futsal match. J Strength Cond Res. 2012;26:2507-14.

8. Kellmann M. Preventing overtraining in athletes in high-intensity sports and stress/recovery monitoring. Scand J Med Sci Sports. 2010;20 Suppl 2:95-102.

9. Montgomery PG, Pyne DB, Hopkins WG, Dorman JC, Cook K, Minahan CL. The effect of recovery strategies on physical performance and cumulative fatigue in competitive basketball. J Sports Sci. 2008;26:1135-45.

10. Rowsell GJ, Coutts AJ, Reaburn P, Hill-Haas S. Effect of post-match cold-water immersion on subsequent match running performance in junior soccer players during tournament play. J Sports Sci. 2011;29:1-6.

11. Borresen J, Lambert MI. The quantification of training load, the training response and the effect on performance. Sports Med. 2009;39:779-95.

12. Johnston RD, Gibson NV, Twist C, Gabbett TJ, Macnay SA, MacFarlane NG. Physiological responses to an intensified period of rugby league competition. J Strength Cond Res. 2012 May 15.

13. Komi PV. Stretch-shortening cycle: a powerful model to study normal and fatigued muscle. J Biomech. 2000;33:1197-206.

14. Horita T, Komi PV, Nicol C, Kyrolainen H. Effect of exhausting stretch-shortening cycle exercise on the time course of mechanical behaviour in the drop jump: possible role of muscle damage. Eur J Appl Physiol Occup Physiol. 1999;79:160-7. 
15. Kellmann M, Kallus KW. Recovery stress questionnaire for athletes: user manual. Champaign: Human Kinetics; 2001.

16. Costa LOP, Samulski DM. Validation process of the recovery-stress questionnaire for athletes (RESTQ-Sport) in portuguese. R Bras Ci Mov. 2005;13:79-86.

17. Coutts AJ, Reaburn P. Monitoring changes in rugby league players' perceived stress and recovery during intensified training. Percept Mot Skills. 2008;106:904-16.

18. Coutts AJ, Wallace LK, Slattery KM. Monitoring changes in performance, physiology, biochemistry, and psychology during overreaching and recovery in triathletes. Int J Sports Med. 2007;28:125-34.

19. Gonzalez-Boto R, Salguero A, Tuero C, Gonzalez-Gallego J, Marquez S. Monitoring the effects of training load changes on stress and recovery in swimmers. J Physiol Biochem. 2008;64:19-26.

20. Nederhof E, Zwerver J, Brink M, Meeusen R, Lemmink K. Different diagnostic tools in nonfunctional overreaching. Int J Sports Med. 2008;29:590-7.

21. Bosco C. La valoración de la fuerza con el teste de Bosco. Barcelona: Paidotribo; 1994.

22. Sattler T, Sekulic D, Hadzic V, Uljevic O, Dervisevic E. Vertical jumping tests in volleyball: reliability, validity, and playing-position specifics. J Strength Cond Res. 2012;26:1532-8.

23. Hopkins WG, Marshall SW, Batterham AM, Hanin J. Progressive statistics for studies in sports medicine and exercise science. Med Sci Sports Exerc. 2009;41:3-13.

24. Horita T, Komi PV, Nicol C, Kyrolainen H. Stretch shortening cycle fatigue: interactions among joint stiffness, reflex, and muscle mechanical performance in the drop jump [corrected]. Eur J Appl Physiol Occup Physiol. 1996;73:393-403.

25. Horita T, Komi PV, Hamalainen I, Avela J. Exhausting stretch-shortening cycle (SSC) exercise causes greater impairment in SSC performance than in pure concentric performance. Eur J Appl Physiol. 2003;88:527-34.

26. Skurvydas A, Brazaitis M, Venckunas T, Kamandulis S, Stanislovaitis A, Zuoza A. The effect of sports specialization on musculus quadriceps function after exercise-induced muscle damage. Appl Physiol Nutr Metab. 2011;36:873-80.

27. Strojnik V, Komi PV. Fatigue after submaximal intensive stretch-shortening cycle exercise. Med Sci Sports Exerc. 2000;32:1314-9.

28. Rowsell GJ, Coutts AJ, Reaburn P, Hill-Haas S. Effects of cold-water immersion on physical performance between successive matches in high-performance junior male soccer players. J Sports Sci. 2009;27:565-73.

29. Ronglan LT, Raastad T, Borgesen A. Neuromuscular fatigue and recovery in elite female handball players. Scand J Med Sci Sports. 2006;16:267-73.

\section{Agradecimentos}

Agradecemos ao CNPQ pela concessão de parte dos recursos que viabilizaram a realização desse estudo (479904/2008 1).

\begin{tabular}{r|r} 
ENDEREço & Fábio Yuzo Nakamura \\
Universidade Estadual de Londrina & \\
Departamento de Educação Física & $\begin{array}{r}\text { Recebido para publicação: 01/04/2013 } \\
\text { Revisado: 25/09/2013 } \\
\text { Grupo de Estudo das Adaptações Fisiológicas ao Treinamento } \\
\text { Rod. Celso Garcia Cid, km 380 } \\
\text { 86051-990 - Londrina - PR - BRASIL } \\
\text { e-mail: fabioy_nakamura@yahoo.com.br }\end{array}$ \\
\hline
\end{tabular}

